\title{
Diffuse versus specular reflection: the influence of hot spots on reflected apparent temperature
}

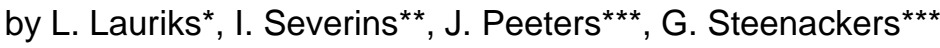

* University of Antwerp, Faculty of Applied Engineering, EMIB research group, Groenenborgerlaan $171-2020$ Antwerpen, Belgium, leen.lauriks@uantwerpen.be

** University of Antwerp, Faculty of Applied Engineering, EMIB research group

*** University of Antwerp, Faculty of Applied Engineering, Op3Mech research group

\begin{abstract}
The reflected apparent temperature is an important factor for performing accurate IR temperature measurements. In this paper, the influence of hot spots on this reflected apparent temperature was investigated for a diffuse versus specular reflector. Two different sizes of hot spots were used, representing a drone battery and an operator. The results were illustrated by IR object temperature readings on two materials (glass and frosted glass) with similar emissivity yet specular versus diffuse reflective characteristics. The results show that hot spots should be avoided at all times, with extra attention when performing spot measurements on short distances and on specular surfaces.
\end{abstract}

\section{Introduction}

Infrared thermography is used for non-destructive analysis in a wide range of applications. All infrared cameras derive the surface temperature from the measured radiation intensity. For this derivation, different parameters should be set correctly in the camera software [1], amongst other the reflected apparent temperature. In this paper, the influence of hot spots in the reflected environment will be investigated: to which extent is the measurement of the reflected apparent temperature influenced by these hot spots, and what is the influence of the object temperature IR measurement? These questions will be investigated under controlled circumstances in laboratory conditions.

\section{Research question}

The reflected apparent temperature can be determined with the reflector method or the direct method [2]. The reflector method is only applicable for places which are accessible to the operator, which is not always the case (e.g. working with a drone at a certain height).

When using the alternative direct method, the operator should choose an incidence angle different from zero (Fig. 1) to avoid reflection of the camera or himself. In some cases, measuring the angles accurately is not possible. This process could be avoided by choosing $0^{\circ}$ for angle $A \& B$, since then only the camera would need to rotate $180^{\circ}$ to measure the reflected apparent temperature. The disadvantage of using $0^{\circ}$ as viewing angle, is the reflection of the operator and/or the equipment itself.

In previous research [3], the effect of the battery heat of a drone on the reflected apparent temperature was investigated. The results showed a rather limited influence of the heated brick on the reflected apparent temperature. The previous results were obtained with a diffuse reflector (wrinkled aluminium foil). However, the results might be different for a specular reflector. The research question for this paper is thus formulated: what is the influence of hot spots on the reflected apparent temperature, and is this influence different for diffuse versus specular reflectors?

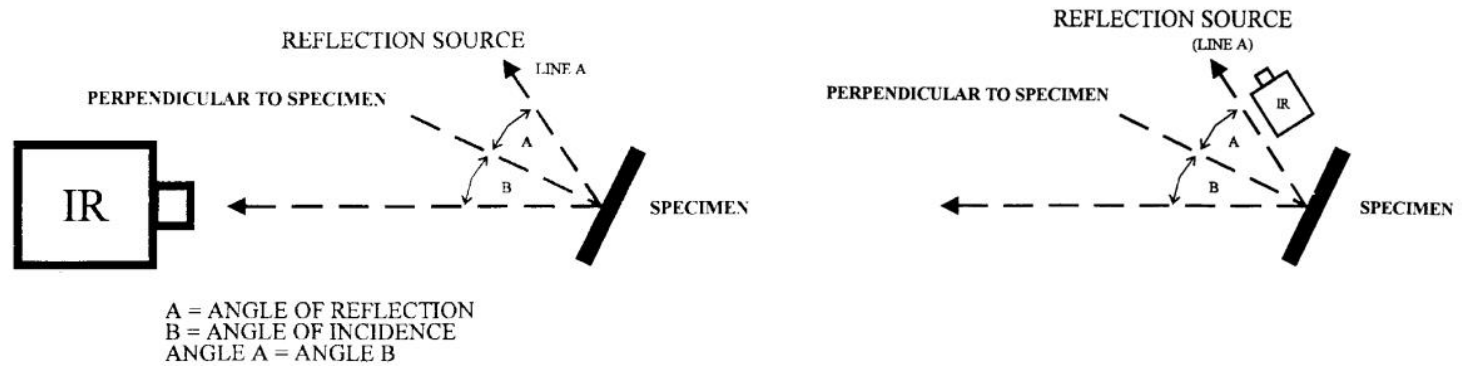

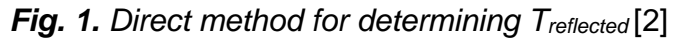




\section{Experimental set-up}

\subsection{Set-up of equipment}

The infrared camera is placed at the end of a corridor, at a fixed spot. The hot spots are positioned next to the camera, at a fixed height. The reflector as well as the objects are mounted on a movable box and can be placed at various distances from the IR camera (Fig. 2). The measured reflected apparent temperature with and without hot spot are compared for the different reflectors and objects.

The experiment is carried out indoors to exclude variations in the surrounding environment (Fig. 3). The set-up is mounted in a corridor with an even distribution of surface temperature (no hot or cold spots). The lighting was switched off before and during the experiment, to avoid hot spots in the ceiling. The average reflected temperature of the environment was measured for each of the 5 studied distances by the reflector method [2]. The results are reported in Table 1.

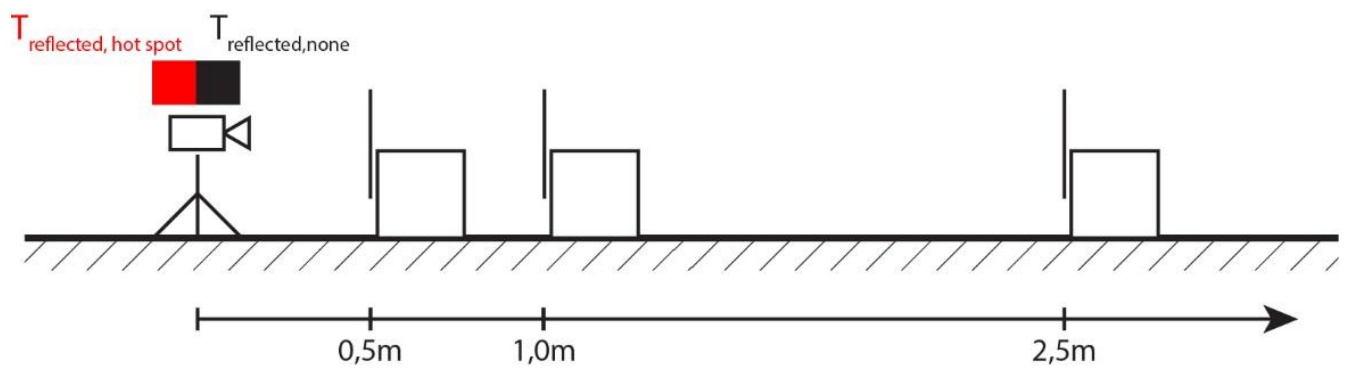

Fig. 2. Schematic representation of measurement set-up
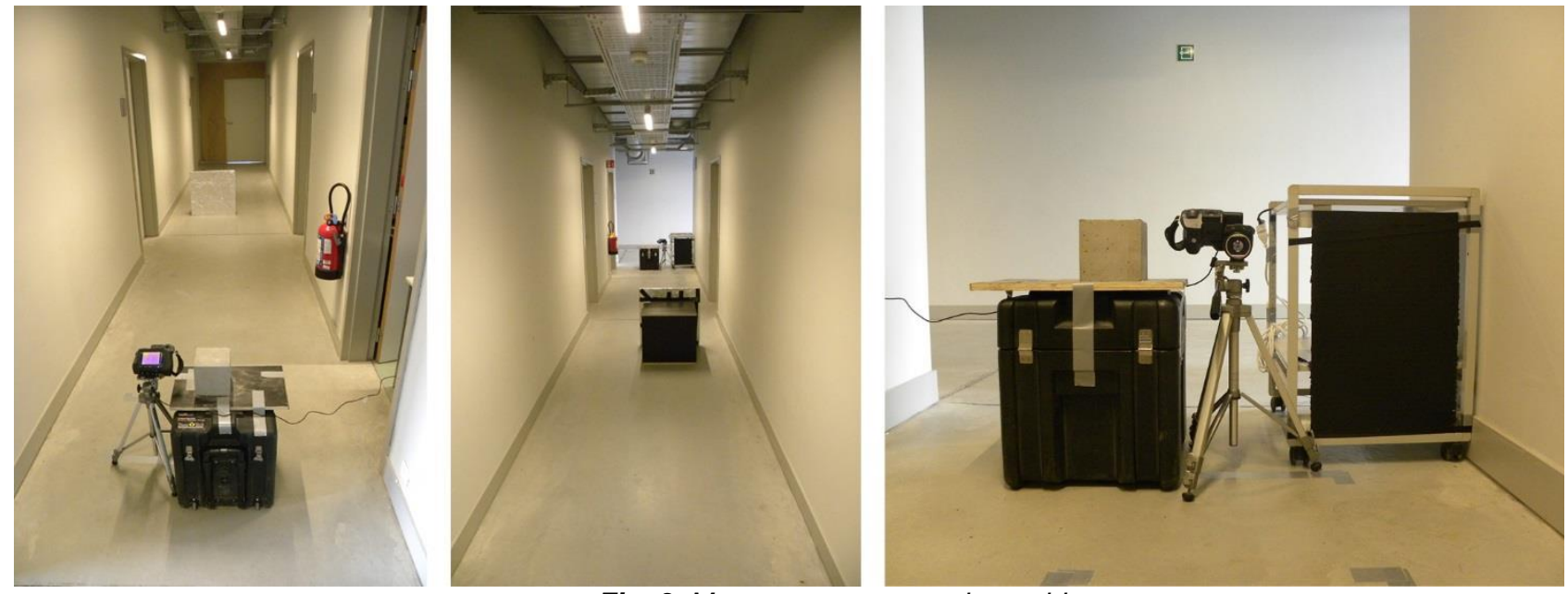

Fig. 3. Measurement set-up in corridor:

(left) view from IR camera to object;

(middle) view from object to IR camera;

(right) positioning of concrete block and heating plate next to IR camera

Table 1. Average reflected apparent temperature of lab environment without hot spots

\begin{tabular}{|l|l|l|l|l|l|}
\hline distance & $\mathbf{0 , 5} \mathbf{~} \mathbf{1 0 , 0}$ & $\mathbf{1 , 0} \mathbf{~}$ & $\mathbf{2 , 5} \mathbf{~}$ & $\mathbf{5 , 0} \mathbf{~}$ & $\mathbf{1 0 , 0}$ \\
\hline $\mathbf{T}_{\text {refl }}$ & $21,1^{\circ} \mathrm{C}$ & $20,8^{\circ} \mathrm{C}$ & $20,7^{\circ} \mathrm{C}$ & $20,6^{\circ} \mathrm{C}$ & $20,5^{\circ} \mathrm{C}$ \\
\hline
\end{tabular}

\subsection{Equipment}

A long-wave microbolometer camera (FLIR T640) is used with a $45^{\circ}$ lens. Dedicated software (FLIR Tools + and Sense Batch) is used for analysing the infrared images.

Environmental conditions in the lab (air temperature and relative humidity) are monitored with a handheld thermoand hygrometer (Metofix TM300). 


\subsection{Hot spots}

Two types of hot spots are investigated in this research. For both hot spots, the aim is to generate a temperature difference of $50^{\circ} \mathrm{C}$ with the reflected apparent temperature of the lab environment of 20 to $22^{\circ} \mathrm{C}$ (Table 1). This difference is based on an outdoor winter scenario, with drone battery or operator between 20 and $30^{\circ} \mathrm{C}$ and a reflected apparent temperature between -30 and $-20^{\circ} \mathrm{C}$.

The first hot spot is an oven heated concrete block of $15 \times 15 \times 15 \mathrm{~cm}^{3}$, which is similar in size to a drone battery. The concrete block is placed in an oven at $80^{\circ} \mathrm{C}$. Preliminary testing revealed that the concrete block's surface temperature remains above $72^{\circ} \mathrm{C}\left(50^{\circ} \mathrm{C}\right.$ above environment $\left.T_{\text {refl }}\right)$ for 7 to 8 minutes.

A heating plate of $60 \times 40 \mathrm{~cm}^{2}$ is used as the second hot spot, to represent an operator. The voltage to the heater is controlled, so that its temperature remains circa $75^{\circ} \mathrm{C}$.

\subsection{Reflectors and objects}

Wrinkled aluminium foil is used as the diffuse reflector. The specular reflector is obtained with the same foil but without wrinkling it.

To illustrate the effect of the hot spots on the infrared object temperature reading, two objects with similar emissivity are compared. Glass was chosen as a specular material. The diffuse material was obtained by sandblasting the glass to obtain frosted glass. The emissivity values of both materials were measured with the noncontact thermometer method, by using black electrical tape (Scotch Super33+, 3M) with an emissivity of 0.95 [4] as the surface-modifying material. The measurements are averaged over 4 or 5 IR pictures of which the results are reported in Table 2.

Table 2. Emissivity measurements of objects

\begin{tabular}{|l|l|l|l|l|l|l|}
\hline Object & $\mathbf{n}^{\circ}$ & $\mathbf{T}_{\text {refl }}$ & $\mathbf{T}_{\text {obj }}$ & $\mathbf{T}_{\text {air }}$ & $\mathbf{R H}_{\text {air }}$ & $\boldsymbol{\varepsilon}$ \\
\hline Glass & 4 & $-58,2^{\circ} \mathrm{C}$ & $11,3^{\circ} \mathrm{C}$ & $-0,9^{\circ} \mathrm{C}$ & $76 \%$ & $\mathbf{0 , 8 4}$ \\
\hline Frosted glass & 5 & $-10,5^{\circ} \mathrm{C}$ & $15,1^{\circ} \mathrm{C}$ & $-1,2^{\circ} \mathrm{C}$ & $49 \%$ & $\mathbf{0 , 8 8}$ \\
\hline
\end{tabular}

\subsection{Experimental procedure}

The experiments are divided in 4 series: one series for each reflector and object. Every series consists of 5 sessions, which leads to 5 pictures for each combination of hot spot, distance and reflector/object. Each session consists of the following steps:

1. Position the reflector/object at the starting distance. Each session uses a different starting distance.

2. Focus the IR camera on the appropriate distance.

3. Take a concrete block out of the oven and place it on the marked spot. Take an IR picture.

4. Position the heating plate at the marked spot, and remove the concrete block out of viewing angle. Take an IR picture.

5. Remove the heating plate out of viewing angle. Take an IR picture without any hot spot.

6. Reposition the reflector/object to the next distance.

7. Repeat steps 2 to 6 until all distances are covered with the same concrete block.

This procedure is carried out with 2 people, and thus takes maximum 6 minutes. The concrete block is thus still above $72^{\circ} \mathrm{C}$ within each session. By alternating the starting distance for each session, possible effects of the concrete block cooling down, are averaged over all distances.

The wrinkled aluminium foil sessions are carried out first. The other three reflector/objects are carried out the next day. The lab environmental conditions during these sessions are listed in Table 3.

Table 3. Environmental conditions in lab during experiment sessions

\begin{tabular}{|l|c|c|c|c|}
\hline Reflectorlobject & Wrinkled alu foil & Flat alu foil & Frosted glass & Glass \\
\hline $\mathbf{T}_{\text {air }}$ & $21,3{ }^{\circ} \mathrm{C}$ & & $21,0{ }^{\circ} \mathrm{C}$ & \\
\hline RH $_{\text {air }}$ & $42 \%$ & \multicolumn{3}{c|}{$41 \%$} \\
\hline
\end{tabular}

\subsection{Image processing}

Due to the time pressure for executing the session with 1 concrete block, some photos were not sharp and were thus excluded from the analysis. The remaining measurements were averaged over the 5 sessions. When 2 or less IR pictures per distance and hot spot remained, this average is left out of further analysis. When 3 pictures remained, this average is marked in grey in the tables showing the results.

For the analysis, a box measurement tool was added to every IR picture, spanning circa the whole reflector/object surface. For these box measurement tools, both the average $\left(T_{\text {avg }}\right)$ and maximum $\left(T_{\max }\right)$ temperature were analysed. 


\section{Results}
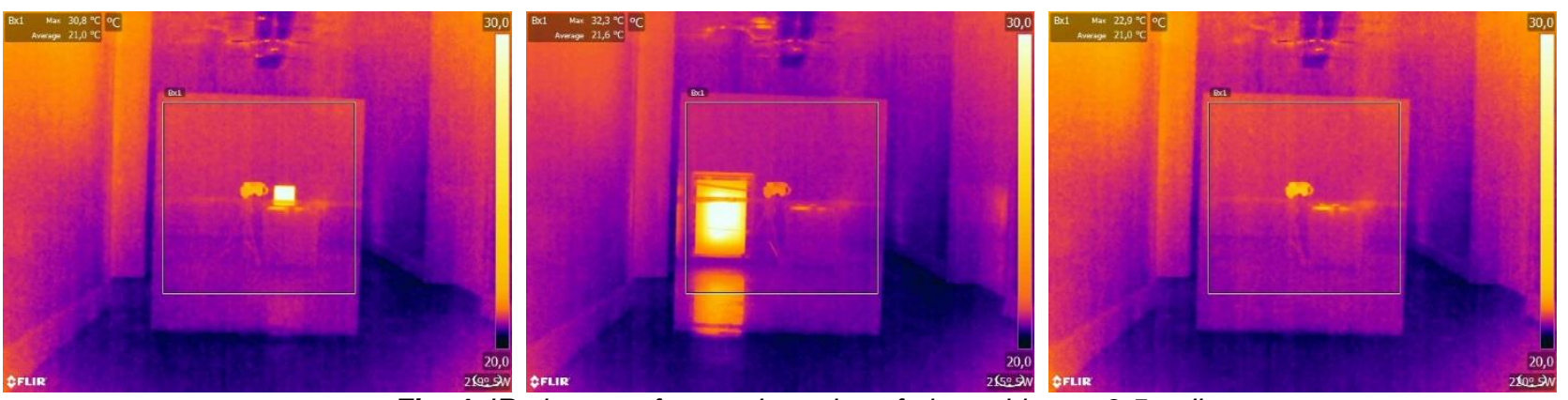

Fig. 4. IR pictures of second session of glass object at 2,5m distance

with (left) concrete block hot spot; (middle) heating plate hot spot; and (right) without hot spot

Table 4. Averaged ( \pm standard deviation) maximum temperature

\begin{tabular}{|c|c|c|c|c|c|c|c|}
\hline & Reflector I & \multirow{2}{*}{\multicolumn{2}{|c|}{\begin{tabular}{|l|l|} 
Heat source & $0,5 \mathrm{~m}$
\end{tabular}}} & \multirow[b]{2}{*}{$1,0 \mathrm{~m}$} & \multirow{2}{*}{$2,5 \mathrm{~m}$} & \multirow[b]{2}{*}{$5,0 \mathrm{~m}$} & \multirow[b]{2}{*}{$10,0 \mathrm{~m}$} \\
\hline & Object & & & & & & \\
\hline \multirow{12}{*}{$\begin{array}{c}\mathbf{T}_{\max } \pm \\
\mathbf{T}_{\max , \text { stdev }} \\
{\left[{ }^{\circ} \mathrm{C}\right]}\end{array}$} & \multirow{3}{*}{$\begin{array}{l}\text { Wrinkled alu } \\
\text { foil }\end{array}$} & concrete block & $69,0 \pm 1,0$ & $63,1 \pm 2,3$ & $35,6 \pm 2,6$ & $24,3 \pm 0,6$ & $20,6 \pm 0,0$ \\
\hline & & heating plate & $67,5 \pm 0,5$ & $64,5 \pm 0,7$ & $42,1 \pm 0,3$ & $31,3 \pm 0,9$ & $24,1 \pm 0,6$ \\
\hline & & none & $46,7 \pm 1,4$ & $36,6 \pm 1,5$ & $23,6 \pm 0,9$ & $20,9 \pm 0,1$ & $20,7 \pm 0,1$ \\
\hline & \multirow{3}{*}{ Flat alu foil } & concrete block & $67,1 \pm 3,5$ & $66,0 \pm 2,1$ & $59,5 \pm 2,6$ & $43,0 \pm 6,9$ & $25,6 \pm 0,8$ \\
\hline & & heating plate & $73,6 \pm 0,6$ & $71,7 \pm 0,4$ & $66,3 \pm 1,4$ & $56,2 \pm 3,4$ & $38,2 \pm 4,3$ \\
\hline & & none & $33,5 \pm 0,9$ & $38,4 \pm 6,5$ & $26,7 \pm 0,6$ & $23,0 \pm 0,9$ & $21,1 \pm 0,1$ \\
\hline & \multirow{3}{*}{ Frosted glass } & concrete block & $21,9 \pm 0,3$ & & $21,6 \pm 0,2$ & $21,3 \pm 0,1$ & $21,2 \pm 0,5$ \\
\hline & & heating plate & $24,7 \pm 0,3$ & & $23,7 \pm 0,3$ & $23,0 \pm 0,4$ & $22,3 \pm 0,2$ \\
\hline & & none & $21,5 \pm 0,2$ & $21,4 \pm 0,1$ & $21,3 \pm 0,1$ & $21,3 \pm 0,1$ & $21,2 \pm 0,1$ \\
\hline & \multirow{3}{*}{ Glass } & concrete block & $31,4 \pm 0,7$ & $31,3 \pm 0,4$ & $31,1 \pm 0,5$ & $31,1 \pm 0,5$ & $30,2 \pm 0,5$ \\
\hline & & heating plate & $32,6 \pm 0,1$ & $32,6 \pm 0,1$ & $32,3 \pm 0,1$ & $32,1 \pm 0,1$ & $31,8 \pm 0,2$ \\
\hline & & none & $25,3 \pm 0,7$ & $23,5 \pm 0,8$ & $22,8 \pm 0,2$ & $22,6 \pm 0,2$ & $22,2 \pm 0,2$ \\
\hline
\end{tabular}

Table 5. Averaged ( \pm standard deviation) average temperature

\begin{tabular}{|c|c|c|c|c|c|c|c|}
\hline & $\begin{array}{l}\text { Reflector I } \\
\text { Object }\end{array}$ & Heat source & $0,5 \mathrm{~m}$ & $1,0 \mathrm{~m}$ & $2,5 \mathrm{~m}$ & $5,0 \mathrm{~m}$ & $10,0 \mathrm{~m}$ \\
\hline \multirow{12}{*}{$\begin{array}{c}\mathbf{T}_{\text {avg }} \pm \\
\mathbf{T}_{\text {avg,stdev }} \\
{\left[{ }^{\circ} \mathrm{C}\right]}\end{array}$} & \multirow{3}{*}{$\begin{array}{l}\text { Wrinkled alu } \\
\text { foil }\end{array}$} & concrete block & $24,0 \pm 0,1$ & $22,1 \pm 0,2$ & $21,2 \pm 0,2$ & $20,8 \pm 0,1$ & $20,4 \pm 0,0$ \\
\hline & & heating plate & $26,7 \pm 0,3$ & $24,4 \pm 0,2$ & $22,7 \pm 0,2$ & $21,7 \pm 0,1$ & $21,0 \pm 0,1$ \\
\hline & & none & $21,1 \pm 0,1$ & $20,8 \pm 0,1$ & $20,7 \pm 0,1$ & $20,6 \pm 0,1$ & $20,5 \pm 0,1$ \\
\hline & \multirow{3}{*}{ Flat alu foil } & concrete block & $21,7 \pm 0,3$ & $21,6 \pm 0,1$ & $21,5 \pm 0,3$ & $21,4 \pm 0,1$ & $21,1 \pm 0,1$ \\
\hline & & heating plate & $27,9 \pm 0,4$ & $25,0 \pm 0,2$ & $24,4 \pm 0,9$ & $23,8 \pm 0,9$ & $22,9 \pm 0,5$ \\
\hline & & none & $21,3 \pm 0,1$ & $21,1 \pm 0,1$ & $20,8 \pm 0,0$ & $20,8 \pm 0,1$ & $20,8 \pm 0,0$ \\
\hline & \multirow{3}{*}{ Frosted glass } & concrete block & $21,4 \pm 0,3$ & & $21,1 \pm 0,1$ & $21,1 \pm 0,1$ & $21,0 \pm 0,1$ \\
\hline & & heating plate & $21,8 \pm 0,2$ & & $21,1 \pm 0,1$ & $21,0 \pm 0,0$ & $21,0 \pm 0,1$ \\
\hline & & none & $21,2 \pm 0,2$ & $21,1 \pm 0,1$ & $21,0 \pm 0,1$ & $21,0 \pm 0,1$ & $20,9 \pm 0,1$ \\
\hline & \multirow{3}{*}{ Glass } & concrete block & $21,8 \pm 0,1$ & $21,3 \pm 0,2$ & $21,2 \pm 0,2$ & $21,1 \pm 0,2$ & $21,2 \pm 0,1$ \\
\hline & & heating plate & $22,6 \pm 0,1$ & $22,0 \pm 0,1$ & $21,8 \pm 0,1$ & $21,8 \pm 0,1$ & $22,1 \pm 0,1$ \\
\hline & & none & $21,2 \pm 0,1$ & $21,2 \pm 0,2$ & $21,1 \pm 0,1$ & $21,2 \pm 0,1$ & $21,0 \pm 0,1$ \\
\hline
\end{tabular}




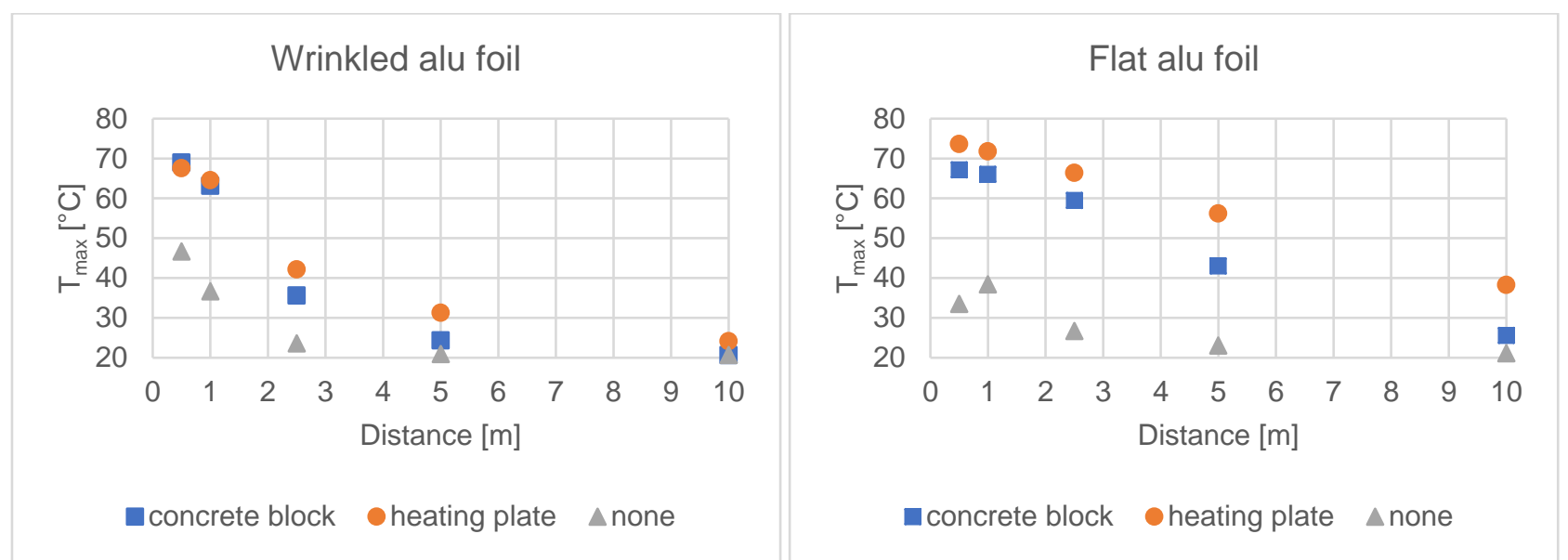

Fig. 5. Tmax graphs for wrinkled and flat aluminium foil

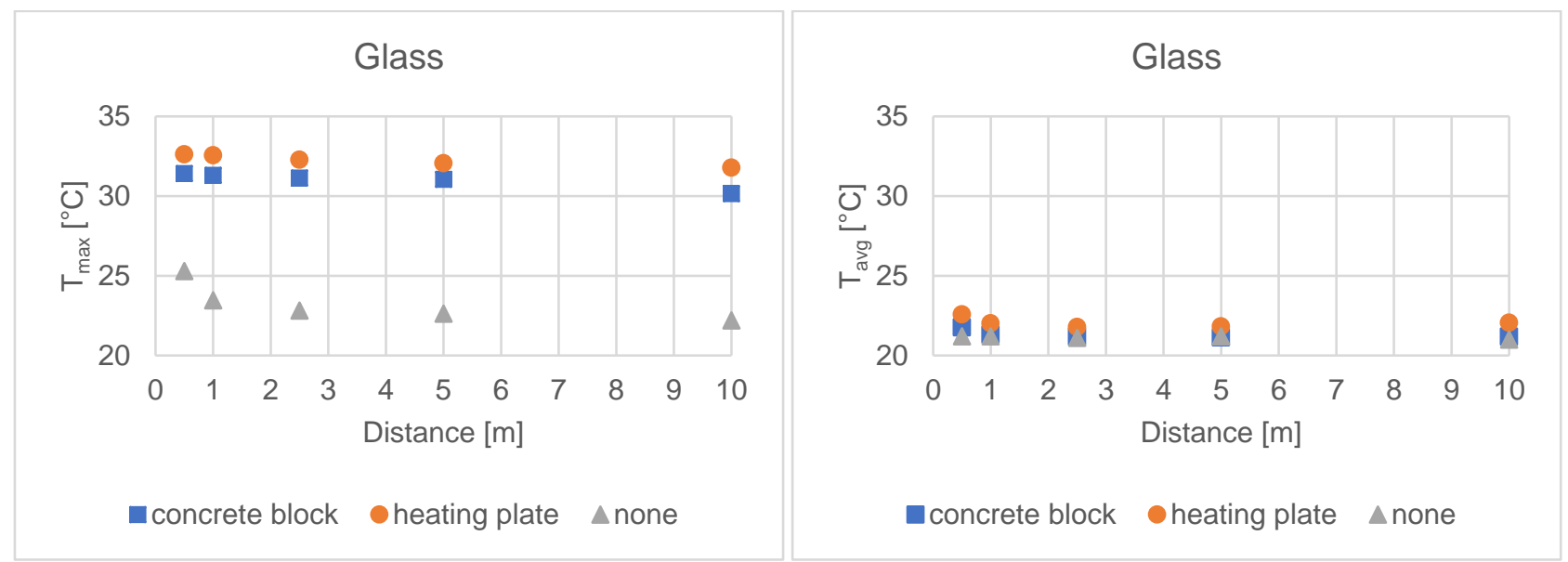

Fig. 6. $T_{\max }$ and $T_{\text {avg }}$ graphs for glass

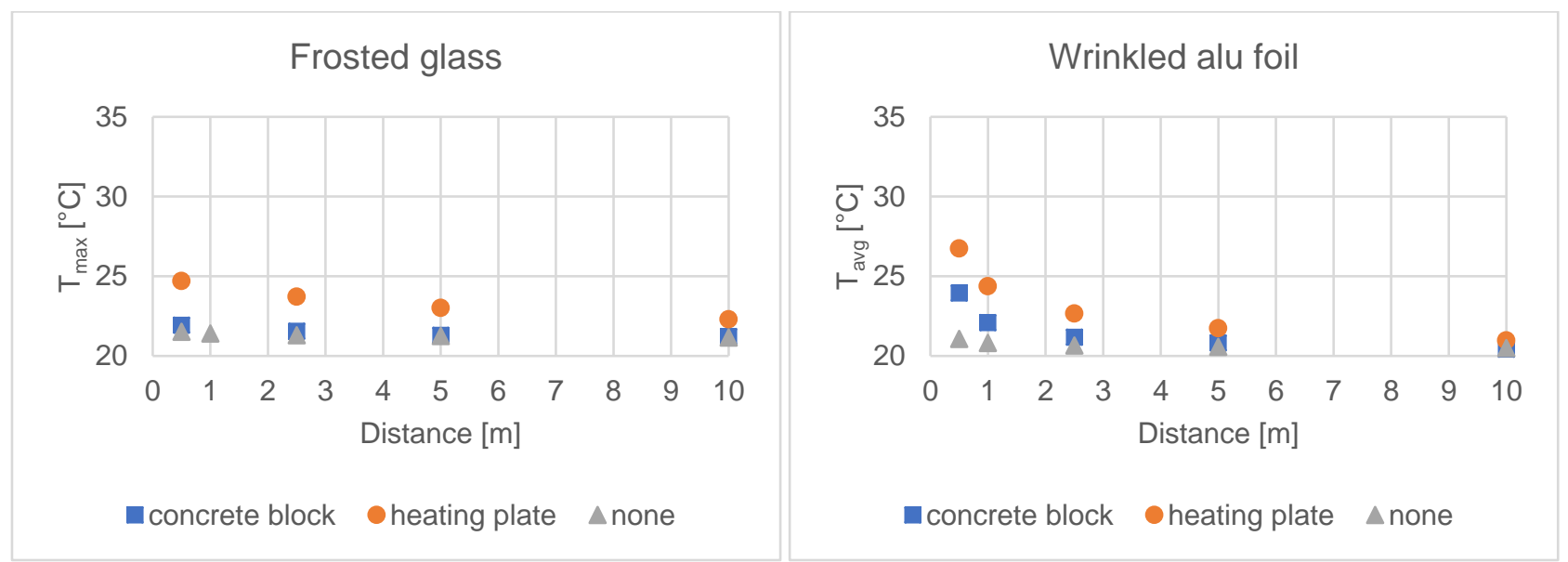

Fig. 7. $T_{\max }$ graph for frosted glass and $T_{\text {avg }}$ graph for wrinkled alu foil

The nominative results are reported in Table 4 and Table 5. Both show the average and standard deviation over all sessions, respectively for the maximum and average temperature of the box measurement tools. Examples of resulting IR picture are shown in Fig. 4. The IR picture on the right, showing the result without hot spot, demonstrates a deviation in the test set-up. The heat from the concrete block is transferred to its support, which is then still present (and thus radiating heat) when taking the picture without hot spot. This influence results in slight increases of temperature readings for the short distances $(0,5$ and 1,0 m) for pictures without hot spots (e.g. Fig. 5 left). These deviations are not considered in the following analysis. 
The standard deviation is analysed for its relevance or relation with other parameters. The standard deviation on the measurements on flat aluminium foil is higher than on the other reflectors/objects. The standard deviation of the maximum temperature is significantly higher than on the average temperature in the box measurement tool. There is no relation between standard deviation on the one hand, and hot spot type or distance on the other hand.

The results of the maximum as well as average temperature in the measurement box tools, are analysed for relations with distance, reflector/object and hot spot type. A selection of graphs is shown in Fig. 5, Fig. 6 and Fig. 7.

A first observation is that the influence of the hot spots decreases with increasing distance (Fig. 5 left). This was expected, since the relative proportion of the hot spot to the environment decreases. The same principle leads to a more prominent influence of the heating plate in comparison with the concrete block, due to its larger size (Fig. 7).

Fig. 5 also shows the difference in influence for diffuse versus specular reflector. The decrease of the influence of the hot spots with increasing distance, is much slower for the specular reflector (Fig. 5 right). The same applies when comparing the specular glass (Fig. 6 left) with the diffuse frosted glass (Fig. 7 left), although the difference is less pronounced.

Another observation considers the difference between the maximum temperature and the average temperature of the measurement box tools. The influence of the hot spots is larger for $T_{\max }$ than for $T_{\text {avg }}$ (Fig. 6). The influence on $T_{\text {avg }}$ can however not be neglected, especially not for the shorter distances (e.g. Fig. 7 right). The influence of the hot spots is the largest on $T_{\max }$ for both wrinkled and flat aluminium foil (cfr. Y-axis scale difference between Fig. 5 and other graphs).

\section{Conclusions}

The influence of the hot spots on the temperature readings, depend on the following parameters:

- $\quad$ the longer the distance between IR camera and measured surface, the lower the influence;

- this decrease per increasing distance, is slower for specular than for diffuse surfaces;

- the larger the hot spots, the higher the influence;

- $\quad$ the influence is higher on spot measurements ( $\left.T_{\max }\right)$ than on averaged readings $\left(T_{\text {avg }}\right)$.

For practical applications, hot spots should be avoided at all times, with extra attention when measuring on short distances and on specular surfaces. The values of spot measurements (e.g. as threshold values) can be influenced even by small hot spots at common distances and even on materials with an emissivity above 0,8 (e.g. $8,4^{\circ} \mathrm{C}$ difference in $\mathrm{T}_{\max }$ between concrete block and without hot spot on glass at $5 \mathrm{~m}$ ).

\section{Acknowledgements}

This research is funded by the University of Antwerp and the Institute for the Promotion of Innovation by Science and Technology in Flanders (VLAIO) via support for the TETRA project "SINT: Smart Integration of Numerical modeling and Thermal inspection", project number HBC.2017.0032.

\section{REFERENCES}

[1] M. Vollmer and K.-P. Möllmann, Infrared thermal imaging: fundamentals, research and applications. Weinheim: Wiley-VCH, 2013.

[2] ASTM American Society for Testing and Materials, "ASTM E1862-97: Standard Test Methods for Measuring and Compensating for Reflected Temperature Using Infrared Imaging Radiometers," 2010.

[3] L. Lauriks, S. Roemendael, G. Steenackers, and A. Audenaert, "Influence of UAV's flight on infrared measurement," in ITC User Conference 2017, 2017, pp. 51-64.

[4] ASTM American Society for Testing and Materials, "ASTM E1993-99a: Standard Test Methods for Measuring and Compensating for Emissivity Using Infrared Imaging Radiometers," 2010. 